\title{
CAR T-cell therapy in multiple myeloma: more room for improvement
}

\author{
Phaik Ju Teoh (i) ${ }^{1,2}$ and Wee Joo Chng (1) $1,2,3$
}

\begin{abstract}
The emergence of various novel therapies over the last decade has changed the therapeutic landscape for multiple myeloma. While the clinical outcomes have improved significantly, the disease remains incurable, typically in patients with relapsed and refractory disease. Chimeric antigen receptor (CAR) T-cell therapies have achieved remarkable clinical success in B-cell malignancies. This scope of research has more recently been extended to the field of myeloma. While B-cell maturation antigen (BCMA) is currently the most well-studied CAR T antigen target in this disease, many other antigens are also undergoing intensive investigations. Some studies have shown encouraging results, whereas some others have demonstrated unfavorable results due to reasons such as toxicity and lack of clinical efficacy. Herein, we provide an overview of CAR T-cell therapies in myeloma, highlighted what has been achieved over the past decade, including the latest updates from ASH 2020 and discussed some of the challenges faced. Considering the current hits and misses of CAR T therapies, we provide a comprehensive analysis on the current manufacturing technologies, and deliberate on the future of CAR T-cell domain in MM.
\end{abstract}

\section{Introduction}

Multiple myeloma (MM), which accounts for $10 \%$ of blood cancers, is a malignancy of plasma cells originating in the bone marrow. Increased understanding of disease biology over the years has led to parallel improvement in treatment modalities, evidenced by the emergence of the novel therapeutics such as proteasome inhibitors (bortezomib, carfilzomib, and ixazomib), immunomodulatory drugs (thalidomide, lenalidomide, and pomalidomide) and U.S. Food and Drug Administration (FDA)-approved monoclonal antibodies (daratumumab and elotuzumab) $)^{1-4}$. While these new therapeutics have led to better disease control, myeloma remains largely incurable, and high-risk patients do not benefit much from this armamentarium of therapies ${ }^{4-7}$. Drug resistance is inevitable and disease relapse remains a great clinical challenge.

\footnotetext{
Correspondence: Wee Joo Chng (mdccwj@nus.edu.sg)

'Department of Medicine, Yong Loo Lin School of Medicine, National

University of Singapore, Singapore, Singapore

${ }^{2}$ Cancer Science Institute of Singapore, Singapore, Singapore

Full list of author information is available at the end of the article
}

Immunotherapy, which was previously deemed a favorable concept, has now evolved into a practical cancer treatment and its progress in the past decade has revolutionized the cancer therapy landscape ${ }^{8,9}$. Chimeric antigen receptor (CAR) T-cell therapy is one of the rapidly emerging and highly promising immunotherapeutic options that has shown unprecedented results in B-cell malignancies ${ }^{10-13}$. It prolongs patients' survival and remission, even for some whom standard treatments have failed $^{14}$.

The success of CAR T-cells therapy in cancer is exemplified by the FDA-approved anti-CD19 CAR T-cell products, namely, tisagenelcleucel/Kymriah, (Novartis) for the treatment of acute lymphoblastic leukemia (ALL) and axicabtagene ciloleucel/Yescarta, (Gilead/Kite) for diffuse large B-cell lymphoma (DLBCL). Most recently (February 2021), CAR $\mathrm{T}$ therapy scene hits another milestone when liso-cel/Breyanzi (Juno/BMS) has also received the FDA-approval for DLBCL treatment, owing to its remarkable efficacy and low incidence of high-grade toxicity $^{15,16}$ (Table 1 and Fig. 1). 
Table 1 FDA-approved anti-CD19 CAR T-cell therapies (true to the time of writing).

\begin{tabular}{|c|c|c|c|}
\hline & Tisa-cel (Kymriah) & Axi-cel (Yescarta) & Liso-cel (Breyanzi) \\
\hline Study & JULIET & ZUMA-1 & TRANSCEND NHL 001 \\
\hline Trial number & NCT02445248 & NCT02348216 & NCT02631044 \\
\hline N (evaluable) & 111 & 101 & 255 \\
\hline Ectodomain & Anti-CD19 & Anti-CD19 & Anti-CD19 \\
\hline Origin of antibody & Murine FMC63 scFv & Murine FMC63 scFv & Murine FMC63 scFv \\
\hline Hinge/Transmembrane & CD8 & CD28 & $\lg G 4$ \\
\hline Endodomain & 4-1BB-CD3乙 & CD28-CD3ろ & 4-1BB-CD3ろ+ eGFRt \\
\hline Genetic manipulation & Lentivirus & Retrovirus & Lentivirus \\
\hline $\begin{array}{l}\text { Starting cells for } \\
\text { manufacturing }\end{array}$ & Bulk T cells & Bulk $T$ cells & CD4+/CD8+ \\
\hline Clinical efficacy & $54 \%$ (CR 40\%) & $83 \%$ (CR 58\%) & $73 \%$ (CR 53\%) \\
\hline Toxicity & CRS: 68\% Neurotoxicity: 14\% & CRS: 94\% Neurotoxicity: 31\% & CRS: $42 \%$ Neurotoxicity: $30 \%$ \\
\hline Survival outcome & $\begin{array}{l}\text { Median PFS: } 3 \text { months Median OS: } \\
8 \text { months }\end{array}$ & $\begin{array}{l}\text { Median PFS: } 8.3 \text { months } \\
\text { Median OS: NA }\end{array}$ & $\begin{array}{l}\text { Median PFS: } 6.8 \text { months Median OS: } \\
21.1 \text { months }\end{array}$ \\
\hline
\end{tabular}

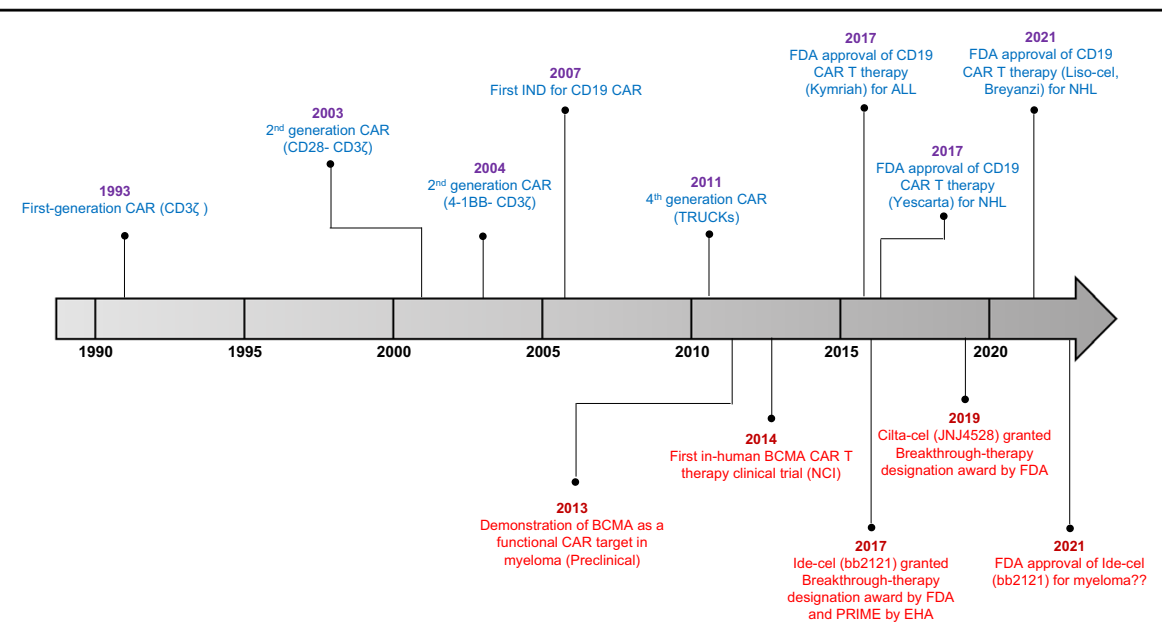

Fig. 1 The time line of CAR T-cell development. The history of CAR T cells and its progress and milestones achieved over the years. Red fonts are the time line for CAR T-cell development in myeloma.

These unprecedented results in B-cell malignancies have spurred a tidal wave of CAR T research in other cancers, including MM. This review details the general development of CAR T-cell therapy and its role in paradigm shift for myeloma treatment. Our topics of discussion range from the current state of its clinical development to the latest technologies adopted in CAR manufacturing for myeloma. Here, we also deliberated on the future perspectives and proposed ways to potentially improve the CAR T-cell research, as we maneuver towards revolutionizing the CAR $\mathrm{T}$ scene in myeloma.

\section{Overview of CAR T-cell therapy}

The history of CAR T-cell therapy dates back to more than two decades ago, when the first ever CAR T cells were created against 2,4,6-trinitrophenyl (Fig. 1). Although an activated immune response was observed, systemic persistence was unfortunately lacking ${ }^{17}$. Subsequent research remained lacklustre until another decade later, the second-generation CAR $\mathrm{T}$ cells directed against CD19 emerged with proven efficacy in preclinical B-cell models ${ }^{18}$. This sets the precedence for the promising venture of CD19-CAR T therapy in ALL and chronic lymphocytic leukemia $(C L L)^{19}$. 
(a)
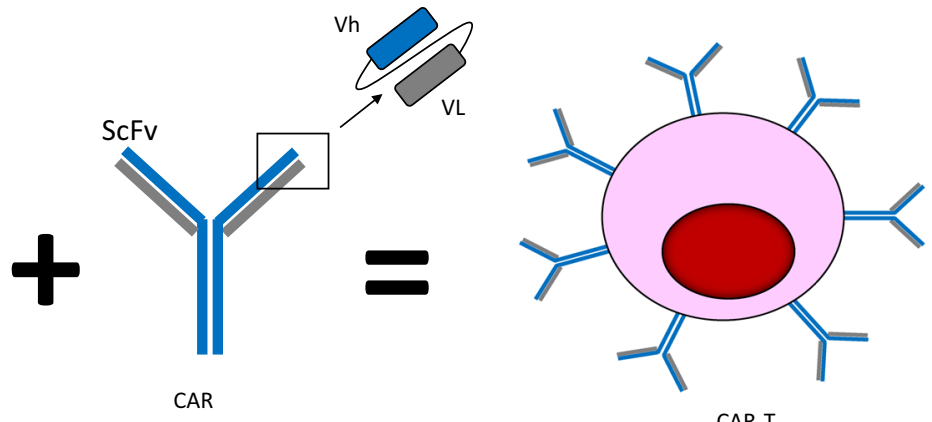

T- lymphocytes

CAR

CAR-T
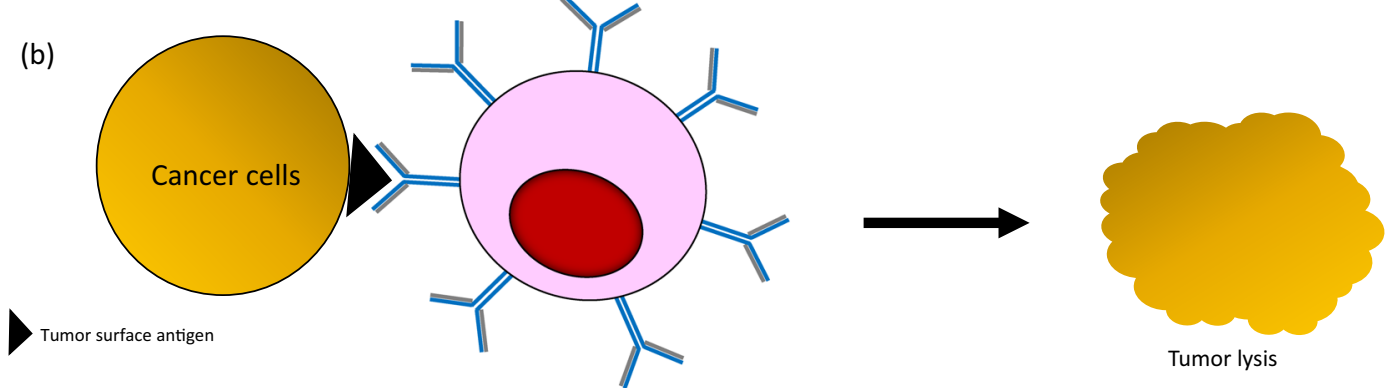

Fig. 2 The basic principle of CAR T-cell therapy. a T lymphocytes are genetically modified to express chimeric antigen receptor which is made up of monoclonal antibody targeting specific antigen of interest. ScFV Single chain variable fragment, Vh Heavy chain, VL Light chain. $\mathbf{b}$ ScFV portion of CAR T cell recognizes tumor-associated antigen on the surface of tumor cells, binds to them, and initiates a cascade of cytotoxic signaling, leading to tumor lysis.

CAR T-cell therapy is able to reprogram host's immune system to attack tumor cells without the physiological need for HLA-presentation. T lymphocytes are biologically engineered to express monoclonal antibodies (moAb) recognizing tumor-associated antigens (Fig. 2). Engagement of these cognate antigens by the antibodybound $\mathrm{T}$ cells will initiate signaling cascades within the $\mathrm{T}$ cells that stimulate release of pro-inflammatory cytokines such as TNF- $\alpha$, IFN- $\gamma$, IL2, and IL6, leading to cytolysis $^{20}$. This unique CAR T-cell property can help mitigate the common limitations of $\mathrm{T}$-cell receptor (TCR)-induced immunity, including the Major Histocompatibility Complex (MHC) loss on tumor cells and low antigen-binding affinity of $\mathrm{T}$ cells ${ }^{21-23}$.

Figure 3 depicts the basic components of a CAR. The single chain variable fragment $(\mathrm{ScFV})$ of a monoclonal antibody on the ectodomain harbors antigen recognition function and is linked to the intracellular domains by a hinge/transmembrane region, commonly derived from CD8 or IgG4. The first-generation CAR $\mathrm{T}$ cell contains only the $\mathrm{CD} 3 \zeta$ signaling domain, which lacks proliferation profile $^{17}$. The current and conventionally produced CAR $T$ products incorporate either one (second-generation) or two (third-generation) co-stimulatory domains (4-1BB, CD28, and/or OX-40) to promote efficient CAR T-cell signaling, persistence, and efficacy ${ }^{24-27}$. The more sophisticated fourth-generation CAR, T-cell redirected for universal cytokine-mediated killing (TRUCK), consists of an added transgene encoding for a pro-inflammatory cytokine, which when induced by signaling molecules, is released to mediate cytotoxicity ${ }^{21,28,29}$.

CAR T-cell manufacturing is a laborious process which takes 2-4 weeks for completion in a Good Manufacturing Practice (GMP) setting (Fig. 4). CAR T-cell therapy is predominantly autologous, and the manufacturing process starts with the collection of peripheral blood mononuclear cells (PBMCs) from patients via leukapheresis. T-cell population is enriched prior to genetic modification with CARs of interest. Gene delivery is performed via viral transduction ( $\gamma$-retrovirus or lentivirus) or non-viral transfer technology (DNA transposon or mRNA transfection) ${ }^{30}$. This is followed by immunophenotyping analyses to ensure successful endowment of $\mathrm{T}$ cells with CARs and cytolytic activities. These CAR $\mathrm{T}$ cells will then undergo ex vivo expansion within a bioreactor, containing growth factor-enriched media, before finally being frozen for storage or immediately transported to the clinic for infusion.

\section{CAR T-cell therapy in MM}

CAR $T$ research in myeloma is still at its infancy, comparatively to lymphoma and ALL (Fig. 1). The most widely studied myeloma CAR target is none other than the $\mathrm{B}$-cell maturation antigen (BCMA), a tumor necrosis factor receptor superfamily member 17 (TNFRSF17). It is 


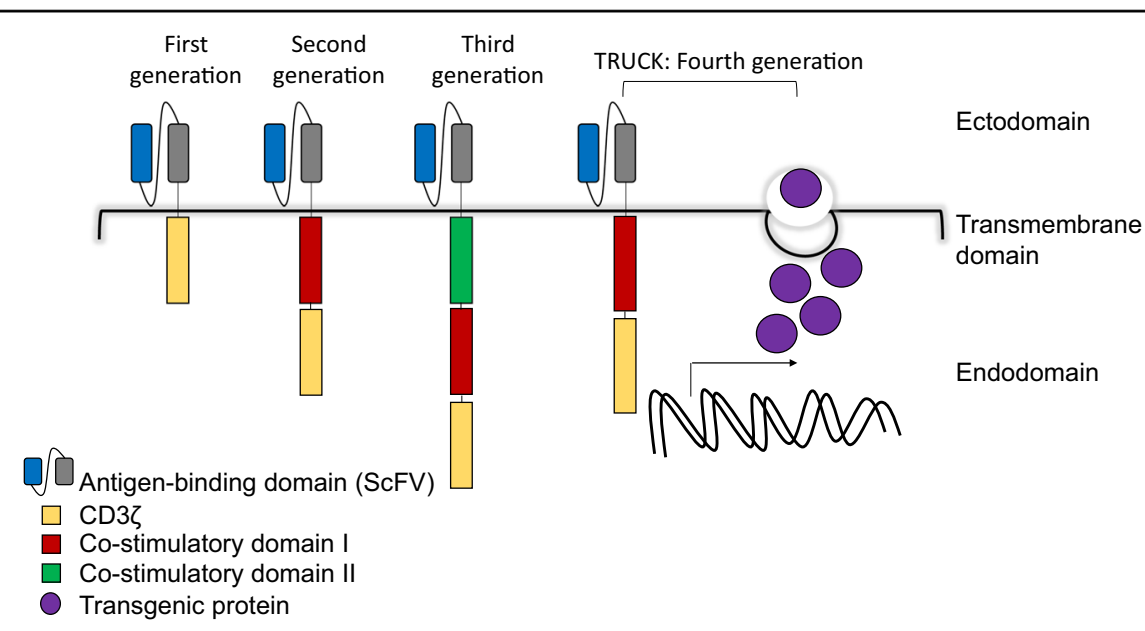

Fig. 3 The depiction of different generations of CAR T cell. First-generation CAR contains basic design with the ScFV portion of monoclonal antibody at the ectodomain, hinge at the transmembrane domain and $\mathrm{CD} 3 \zeta$ signaling molecule at the endodomain. Second- and third-generation CARs comprise additional one or two co-stimulatory molecule/s in the endodomain, respectively. Fourth-generation CAR (TRUCK) follows the basic design of second-generation CAR, coupled with an added transgene encoding for a cytokine (transgenic protein).
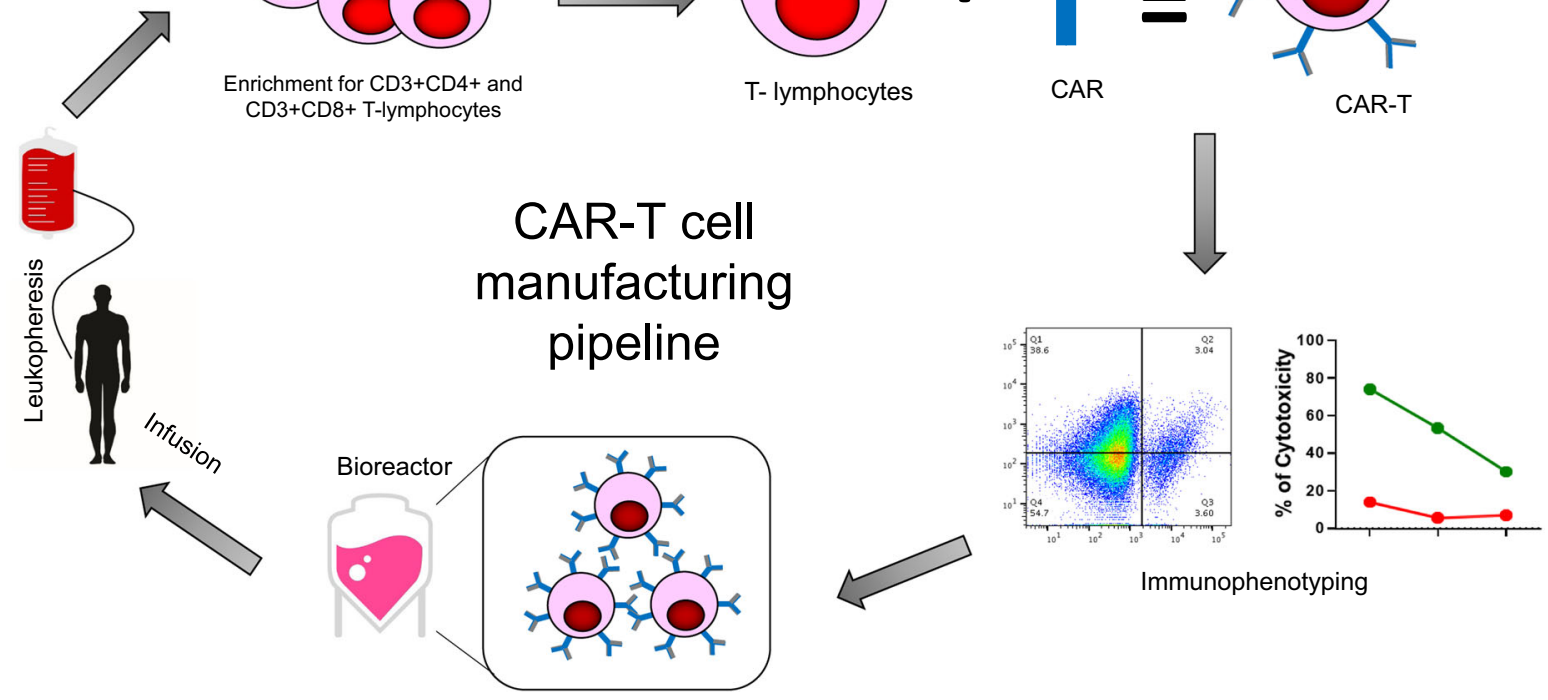

Immunophenotyping

Fig. 4 CAR T-cell manufacturing pipeline. The process starts off with leukapheresis to obtain PBMCs from the patients. Enrichment of T cells is done prior to genetic modifications via viral or non-viral gene delivery system. This is followed by immunophenotyping analysis to determine the cytokine and cytolytic profile of the CAR T cells. Positive CAR T cells are selected to undergo ex vivo expansion in a bioreactor vessel containing growth factor-enriched media. When the required amount is obtained, the CAR T cells will be transported back to the clinic for patient infusion.

deemed an ideal antigenic target due to its preferential expression on the plasma cells but not on hematopoietic stem cells ${ }^{31}$. Binding and stimulation of ligands, B-cell Activating Factor (BAFF) and A Proliferation-Inducing Ligand (APRIL), to BCMA promotes growth and proliferation of plasma cells in the bone marrow. Although the expression of BCMA is heterogenous ${ }^{32,33}$, it is universally present in all MM cells ${ }^{31,34}$ and its overexpression carries important prognostic value ${ }^{35,36}$.

The first BCMA-directed CAR was developed less than a decade ago, showing preclinical evidence of functional targetability ${ }^{31}$. This was followed by the first-in-human 
Phase I clinical trial to test the efficacy of the BCMAtargeted CAR $\mathrm{T}$ cells in relapsed/refractory multiple myeloma (RRMM) (NCT02215967). The overall response rate (ORR) was a good $81 \%$, with $63 \%$ demonstrating very good partial (VGPR) or complete response $(\mathrm{CR})^{37,38}$. Consequentially, there was a widespread effort to develop novel anti-BCMA CARs and to fine-tune the old CARs.

Table 2 documents the anti-BCMA CAR T-cell clinical trial records, with the latest updates from $\mathrm{ASH}$ $2020^{38-40}$. The first significant breakthrough in CAR Tcell development for myeloma was reached when bb2121 (Bluebird Bio) was granted a breakthroughtherapy designation award by the FDA and Priority Medicine (PRIME) eligibility award by the European Medicines Agency (EMA) in 2017. This was precipitated by its excellent efficacy and durable responses. Latest reported ORR was $73 \%$ with significantly improved PFS (median PFS 8.8 months) in the RRMM patients ${ }^{41,42}$. Bb2121 has been heralded as the latest front-runner for FDA's approval for MM treatment. Its successor bb21217, designed with the same CAR construct but is being cultured in the presence of PI3K inhibitor bb007 ex vivo, has recorded a decent ORR of $55 \%$, associated with enrichment of memory $\mathrm{T}$-cell phenotype and high-peak expansion ${ }^{43}$. Additionally, JNJ-4528, the bi-epitope CAR T-cell product containing two BCMA-targeting domains, aimed to enhance the antigen binding affinity, has also shown an impressive 94.8\% ORR in the CARTITUDE- 1 trial $^{44,45}$. This study provides compelling evidence that a single low-dose infusion of the CAR $\mathrm{T}$ product was sufficient to induce early, deep and durable response. JNJ-4528 takes after the exact construct of LCAR-B38M that is being evaluated in another geographical cohort (China) in the LEGEND-2 trial. The study reported a promising ORR of $82.4 \%$ with a good safety profile in the high-risk patient subgroup ${ }^{46}$.

Some other noteworthy BCMA-CAR T products are the human-derived and fully humanized CARs (JCARH125, MCARH171, and FCARH143), designed to reduce the graft-versus-host disease (GVHD) and to prolong the persistence of the $\mathrm{T}$ cells ${ }^{47}$. ORRs to these treatments were a remarkable 64,100 , and $82 \%$, respectively. The efficacy of JCARH125, in particular, was reported to be associated with T-cell product that is of high CD3+ purity and enrichment of early-memory phenotype and polyfunctionality ${ }^{48}$.

Described in Table 2 are many more BMCA CAR T trials that are ongoing and recruiting, with different products demonstrating different levels of efficacy. A recent meta-analysis study on a total of 23 different BCMA-CAR T-cell products that have been used in a total of 640 patients, reported an average ORR of $80.5 \%$, with $44.8 \%$ $\mathrm{CR}$ and 12.2 months median $\mathrm{PFS}^{49}$.

\section{Limitations to BCMA-CAR-T therapy}

Alas, the promising results from anti-BCMA CAR T-cell therapies do not come without its own set of challenges.

\section{Therapeutic resistance}

The underlying mechanisms for therapeutic resistance have remain largely unclear, but tumor heterogeneity, and antigen escape have been implicated ${ }^{50}$.

Heterogeneously expressed BMCA at the intra-tumor level can lead to preferential targeting of cells with high BCMA while sparing those with low/zero BCMA expression, resulting in the outgrowth of the latter clones $^{33,34,47}$. Indeed, BCMA often loses its expression upon disease relapse after the first CAR $\mathrm{T}$ infusion, suggesting the selection for BCMA-negative MM clones by the CAR T cells ${ }^{38,51-53}$.

For BCMA antigen escape, one of the most welldescribed ways is the erroneous physiology of the BCMA antigen. BCMA can be inadvertently transferred from tumors to $\mathrm{T}$ cells in a process called trogocytosis, causing $\mathrm{T}$-cell fatricide ${ }^{3,50}$ or it can be shed into the blood circulation (now called serum BCMA (sBCMA)), mediated by $\gamma$-secretase ${ }^{47}$. Both can cause dampening of tumor cell recognition $^{38,54}$. Although lower sBCMA level is indeed associated with good $\mathrm{ORR}^{36,38,55,56}$, it does not always correlate with CAR $\mathrm{T}$ dose-response and its expression remains low at the time of relapse with increase disease burden $^{52}$, indicating that mechanism of resistance can extend beyond tumor intrinsic factors.

In relation to this, molecular BCMA aberration was recently highlighted by a study using longitudinal singlecell transcriptomic and bulk genomic analysis. They identified that resistance to bb2121 in a patient was associated with biallelic loss of BCMA. Majority of the MM cells demonstrated heterozygous deletion of chr16p, containing the BCMA locus, concomitantly with nonsense mutation of the other allele, rendering a biallelic inactivation of $\mathrm{BCMA}^{52}$. This was corroborated by another study which revealed an association of genomic instability with biallelic deletion of BCMA loci upon CAR $\mathrm{T}$-cell infusion ${ }^{51}$. Both alleles were intact before CAR $\mathrm{T}$ treatment, therefore, suggesting the occurrence of branching evolution over treatment course and acquisition of genomic aberrancy by MM cells in selecting for BMCA-negative cells.

\section{Toxicity}

Toxicity such as cytokine release syndrome (CRS) and neurotoxicity, mediated by pro-inflammatory cytokines, upon CAR T-cell activation, is another persistent problem. CRS manifests as fever, nausea, and flu in mild cases, but could escalate to hypotension, cardiac arrest, and liver failure in severe cases. On the other hand, neurotoxicity could range from mild confusion and delirium, to severe 


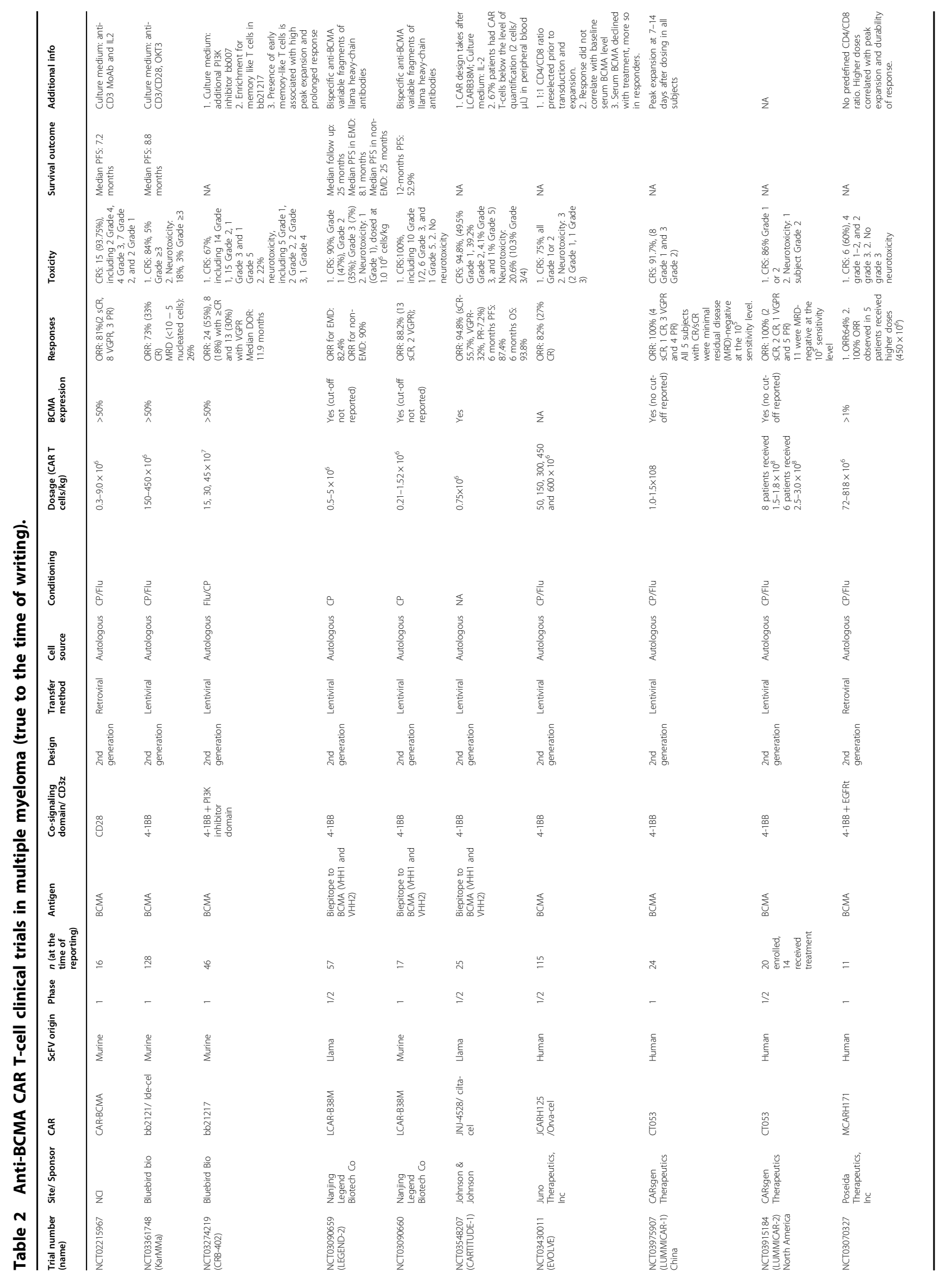




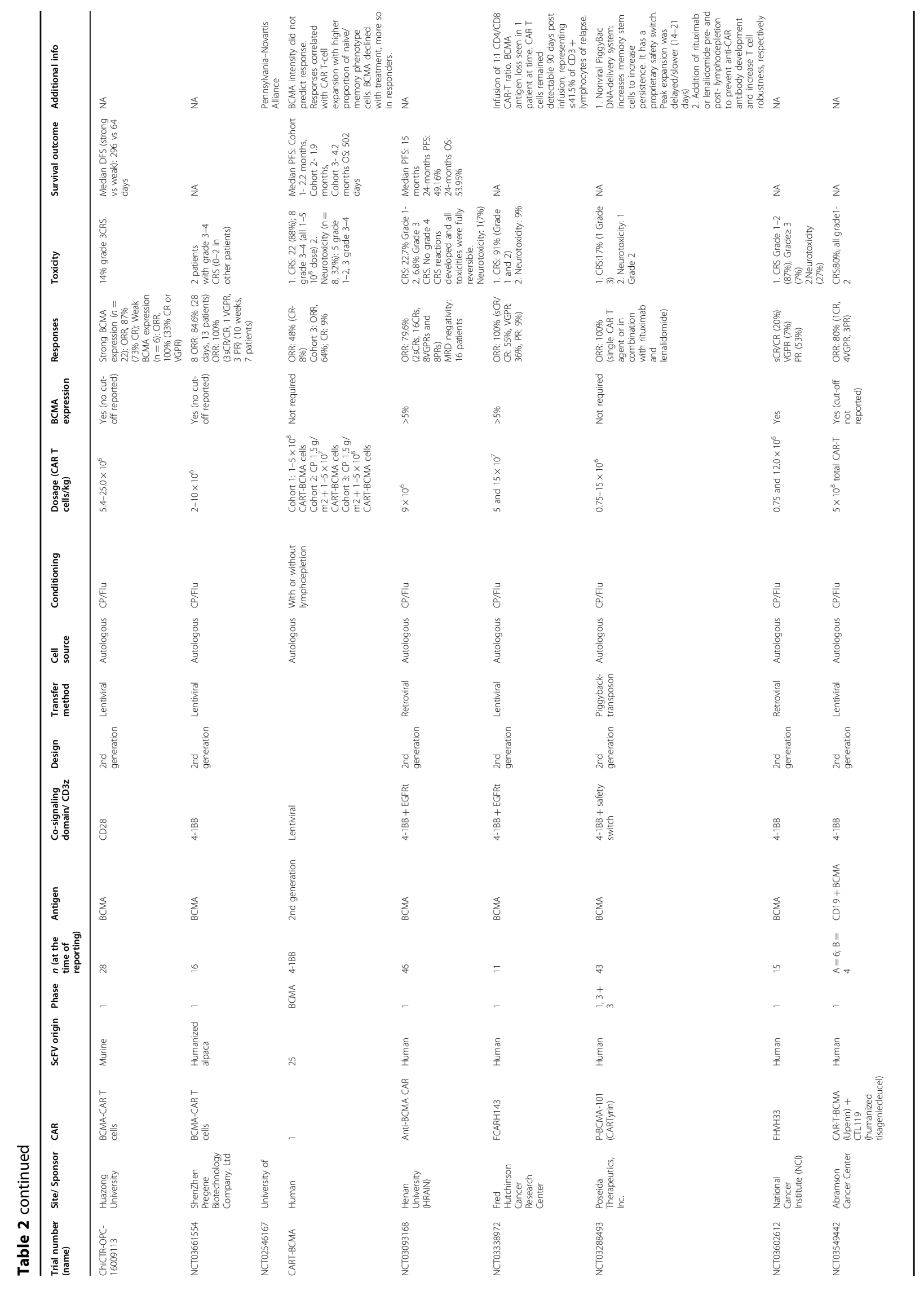




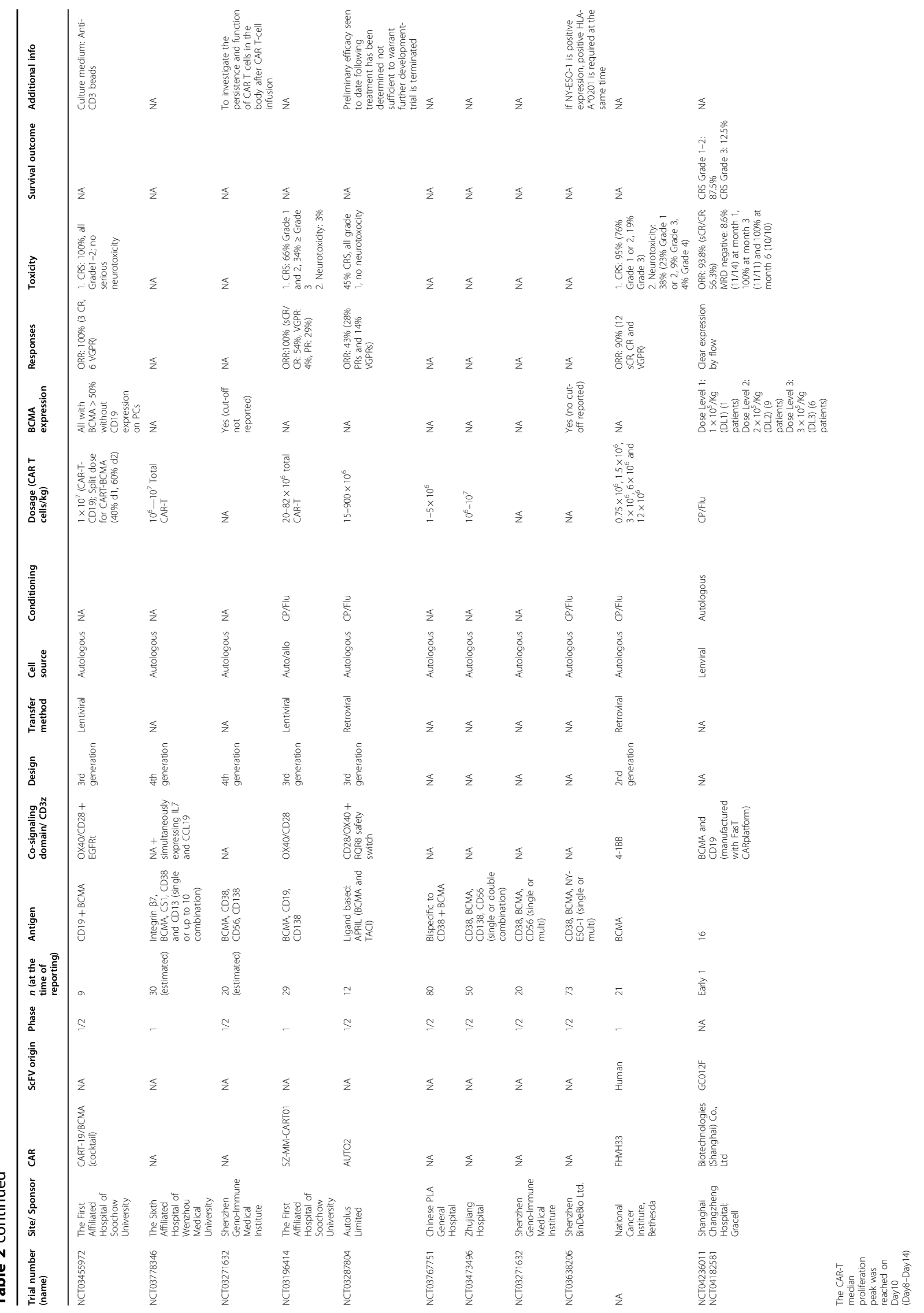




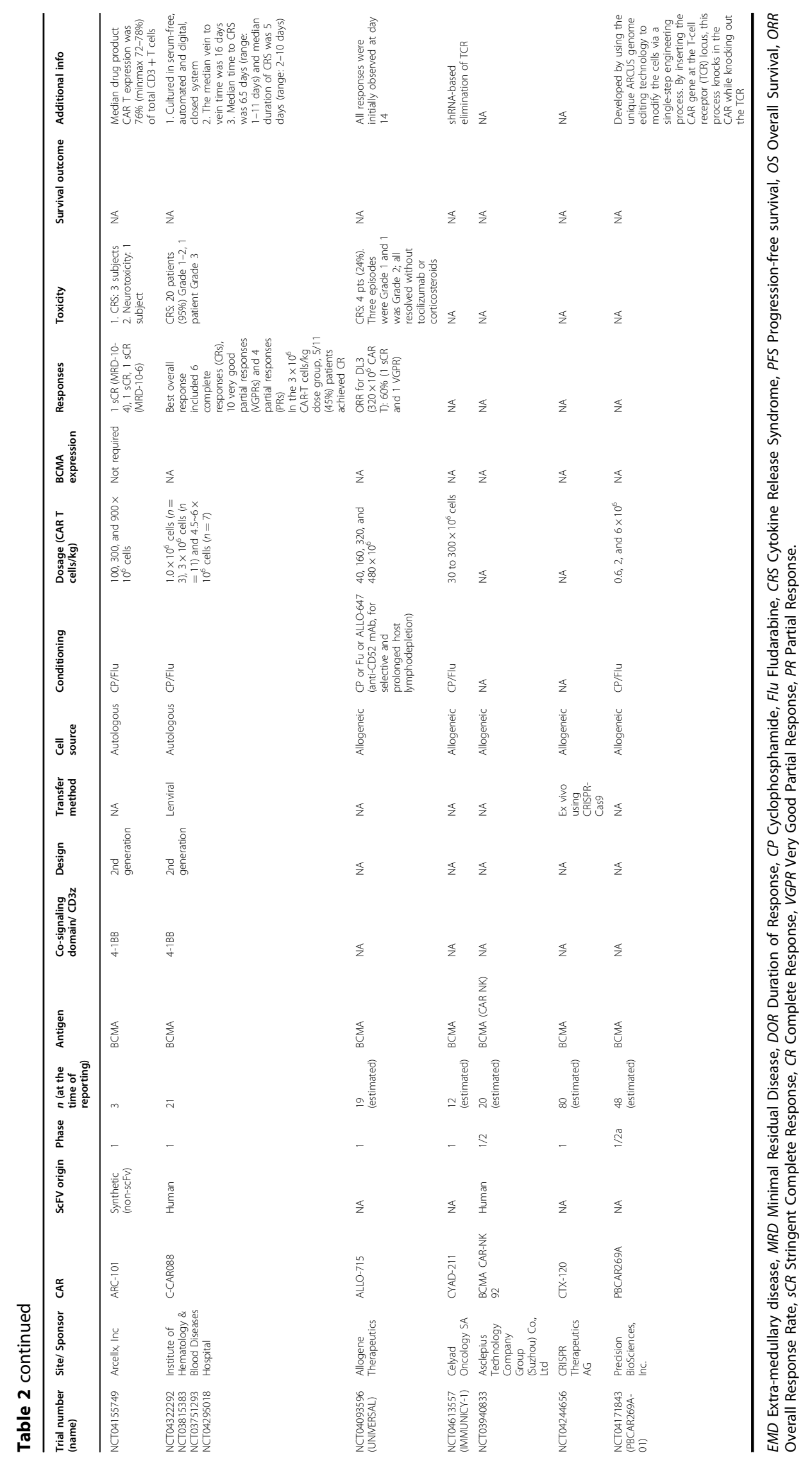


obtundation, seizure, and white matter degradation ${ }^{20}$. Despite being a plasma cell marker, BCMA is also coexpressed on normal B-lymphocytes, therefore, BCMA CAR T-cell therapy could also introduce on-target/offtumor effects, where the common manifestations include B-cell aplasia, neutropenia, and immunosuppression that leads to increased infection risks. In the same aforementioned meta-analysis study, an average CRS and neurotoxicity prevalence of $80.3 \%$ and $10.5 \%$, respectively, were reported $^{49}$. While toxicities are frequent events, toxicityrelated lethality in the patients are controllable. High-grade toxicities are more often observed in patients with heavy tumor burden or on high CAR T-cell dosage ${ }^{38}$. Toxicities are typically managed with readily available drugs such as IL6R-antagonist (tocilizumab) for CRS, and corticosteroids for neurologic symptoms ${ }^{20}$.

\section{Non-BCMA CAR T cells}

The emergence of next breakthrough molecule is perpetually anticipated. Various surface antigens have been explored in this pursuit. This section documents the progress made by some of the more well-studied myeloma antigens (Table 3).

\section{SLAMF7/CS1}

The attractiveness of employing signaling lymphocytic activation molecule F7 (SLAMF7) for CAR targeting stems from elotuzumab, the first SLAMF7 humanized moAb that was FDA-approved for RRMM ${ }^{57,58}$. Anti-SLAMF7 CAR $\mathrm{T}$ cells deriving from elotuzumab showed preclinical evidence of rapid cytolysis in primary myeloma samples and elimination of extramedullary cells in xenografts ${ }^{59}$. SLAMF7-CAR T cells incorporating on/off suicide gene for enhanced safety and tolerability was later developed for clinical investigations (NCT03958656 and NCT03710421). A European group has also engineered a novel SLAMF7 CAR T-cell model utilizing non-viral gene transfer approach of the Sleeping Beauty transposon system. Their proprietary product is currently being tested in Phase I/II CARAMBA trial (https://www.caramba-cart.eu/patients/) ${ }^{60}$.

Signifying a milestone, the allogenic anti-SLAMF7-CAR T cell (UCARTCS1) became the first 'off-the-shelf' CAR T-cell product in MM gaining FDA-approval for clinical trial (MELANI-01). UCARTCS1 was manufactured using healthy allogenic $\mathrm{T}$ cells with the TALEN gene editing technology to eliminate endogenous TCR and SLAMF7 expressions, intended to reduce the risk of GVHD and T-cell fratricide ${ }^{61}$.

Importantly, a novel anti-SLAMF7/BCMA bispecific CAR T-cell product is under preclinical development with aim of increasing tumor coverage and overcoming antigen loss. These CAR $\mathrm{T}$ cells with single chain antibody containing two ligand-binding domains (one for SLAMF7 and another for BCMA) connected in tandem, showed enhanced activity, compared to $\mathrm{T}$ cells expressing individual CARs. In combination with PD1 checkpoint inhibitor, it confers accelerated tumor clearance in vivo ${ }^{62}$.

\section{CD38}

The clinical success of anti-CD38 moAb (daratumumab and the second-generation isatuximab) in $\mathrm{MM}^{63-65}$, underlies the development of anti-CD38 CAR T cells. Preclinical evidence of effective anti-myeloma effects of anti-CD38 CAR T-cell therapy ${ }^{66,67}$ has led to the initiation of various clinical trials, one of which investigates anti-CD38 CAR $\mathrm{T}$ cells as monotherapy in RRMM (NCT03464916), while others explore its combination with different CAR $\mathrm{T}$ cells, including anti-BCMA, antiCD19, anti-CD138, anti-CD56, and anti-NY-ESO- ${ }^{3}$. A dual-specificity anti-CD38/BCMA CAR T-cell product is being evaluated (NCT03767751).

It is noteworthy, however, that CD38 is generally not highly expressed on myeloma cells and its expression can be downregulated in advanced disease; ${ }^{67,68}$ thus, resistance to the anti-CD38-CAR-T may be expected. There is also a likelihood for on-target/off-tumor toxicity as CD38 is also expressed on activated $\mathrm{T}$ cells (thereby, increasing the risk of T-cell fratricide), NK cells and normal prostate, neuronal, and muscle cells.

\section{CD19}

Although CD19 is typically absent in matured plasma cells, minor subsets of myeloma cells with unique propagating properties express low CD19, associated with drug resistance and relapse-promoting properties ${ }^{3,54}$, making it a plausible therapeutic target. A proof-of-concept pilot study was conducted with anti-CD19 CAR T cells, Kymriah. Out of 12 patients treated, six achieved VGPR, two achieved PR, and two other experienced progressive disease (NCT02135406) $)^{13}$. Importantly, a cocktail CAR T product of anti-CD19/anti-BCMA produced an impressive 100\% ORR (NCT03455972). Despite more than 1000-fold cell expansion at the peak data-point, patients experienced only mild (Grade 1-2) CRS and no neurotoxicity.

\section{TACI}

Transmembrane activator and CAML interactor (TACI), like BCMA, is a member of the TNF superfamily ${ }^{34}$. Both TACI and BCMA share the same activating ligands, APRIL and BAFF, which when bound to their receptors, confer MM growth and survival. Based on this biological nature, ligand-based CAR T cells directed against APRIL have been developed to concurrently target both the TACI and BCMA signaling pathways ${ }^{69,70}$. In an in vivo model, APRILdirected CAR $\mathrm{T}$ cells eradicated $\mathrm{BCMA}^{+} \mathrm{TACI}^{-}$and $\mathrm{BCMA}^{-} \mathrm{TACI}^{+}$tumors, but monospecific anti-BCMA CAR $\mathrm{T}$ cells failed to contain the proliferation of the $\mathrm{BCMA}^{-}$ cells, suggesting the potential application of APRIL-directed CAR $T$ cells in cases associated with BCMA loss ${ }^{69}$. More 


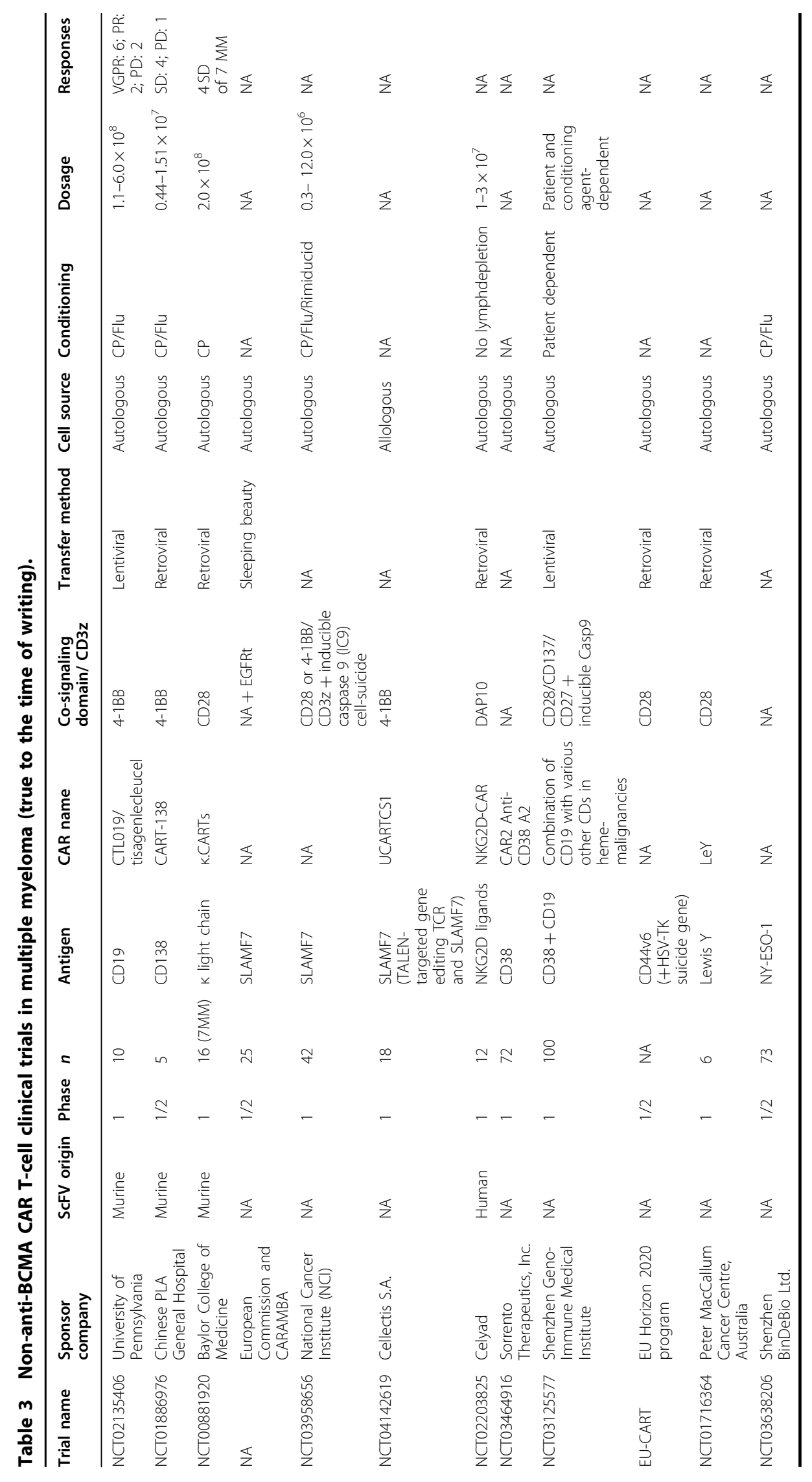


recently, another APRIL-targeting CAR T-cell product with a refined molecular design has been reported ${ }^{70}$. This trimeric APRIL-based CAR T cells have enhanced binding to BCMA and TACI receptors with increased cytolytic activities, compared to its monomeric counterpart. Despite these promising preclinical data, the only clinical trial on APRILtargeting CAR $\mathrm{T}$ cells conducted till date was terminated due to insufficient efficacy (NCT03287804).

There is only one reported CAR T-cell product in development that specifically targets TACI surface antigen. It is the bispecific TACI/BCMA CAR $\mathrm{T}$ cells, that possess efficient in vitro and in vivo cytotoxicity towards MM cells ${ }^{71}$.

Besides myeloma cells, TACI is also found on the immunosuppressive regulatory $\mathrm{T}$ cells (Tregs). Targeting TACI, thus, may not only cause cytotoxicity through direct cytolysis, but also by indirectly manipulating the hostile microenvironment imposed by Tregs $^{34,72}$, rendering a good two-pronged approach.

\section{CD138}

CD138 is a highly expressed surface molecule on MM cells, used clinically as a selection marker for plasma cells. The Chinese PLA Hospital tested a second-generation 41BB/CD3 CD138-targeted CAR T-cell product as a monotherapy in MM; however, only modest therapeutic response was reported (NCT01886976). This data was obtained from a small sample size $(n=5)$, thus results may not be sufficiently powered for meaningful interpretation. Anti-CD138 CAR $\mathrm{T}$ cells have also been incorporated into other CAR T-cell cocktail (BCMA, CD38, CD19, SLAMF7) trials but no substantial data can be obtained (NCT03778346 and NCT03196414).

Despite its overexpression in myeloma cells, it should be noted that CD138 is present in other normal tissues such as epithelial, endothelial, and vascular smooth muscle cells, again, underlining the possibility of on-target/off-tumor effects. This drawback was evident in past clinical trials investigating the anti-CD138 antibody-drug conjugate for MM treatment where some patients reported serious mucosal and skin toxicity ${ }^{13}$.

\section{Others}

Amidst the intensive research on CAR T-cell therapy, various other target antigens have also been examined, some of the worth mentioning ones are G-proteincoupled Receptor Class C Group 5 Member D (GPRC5D), CD56, Lewis Y, CD44v6, and Kappa-light chain. These molecules were studied because of their surface expression and prognostic implications ${ }^{66}$. Comprehensive data from trials of these CAR T-cell products are mostly unavailable for evaluation but for some that have trial data released, there is not much excitement as the patients reported modest to low responsiveness ${ }^{54}$.
Various other molecules, such as CD229, integrin $\beta 7$, CD70, and CD126 (IL6R) involved in plasma cell biology are being investigated preclinically ${ }^{3,73}$.

\section{Technological advances of CAR T-cell manufacturing in myeloma}

As CAR T-cell therapies continue to evolve, multimodal engineering of effector cells offers the prospect of tackling increasingly complex disease settings. To address the issue of antigen escape upon disease relapse, CARs targeting double epitopes of BCMA have been explored. LCAR-B38BM and JNJ-4528 were built to express two BCMA-binding domains to increase its binding affinity to low BCMA-expressing myeloma cells. The caveat to this approach, however, is that there is yet a conclusive answer as to whether a certain threshold of BCMA expression is required for optimal tumor recognition and cytotoxicity. The study by University of Pennsylvania (NCT02546167) revealed no correlation between baseline BCMA expression and the response rate $;^{74}$ in contrast, the FCARH143 trial (NCT03338972) found differential BCMA expression on tumor cells of long-term responders versus relapse patients prior to the treatment. In the many $\mathrm{BCMA}$ clinical trials conducted till date, the baseline BCMA is or is not an inclusion criterion and if it is, the range encompasses a mere observable expression $(\sim 1 \%)$ to a high $>50 \%$ (Table 2). This anticipated inter-study heterogeneity makes it difficult to evaluate the potential factors contributing to safety and efficacy. A single clinical trial that sets a range of different baseline levels may be able to inform on the antigen threshold required for desired anti-myeloma effect and if a lower baseline BCMA confers more rapid resistance/relapse.

In overcoming clonal selection for single antigen loss, dual/multi-antigen targeting has been approached by either (a) infusing patients with multiple CAR T-cell products in a cocktail regimen or (b) having double ScFVs for different antigens within one $\mathrm{T}$ cell. Some of the known antigens that are being co-expressed with BCMA CAR are SLAMF7, CD19, and CD38 (Table 2). This strategy is pertinent considering these targets have remained stably expressed at progression in BCMAnegative setting ${ }^{51}$. However, it is crucial to consider the accompanying challenges. For example, in (b), controlling the ratio of positivity of each CAR within the T cells may be technically challenging. The difficulty to define the expression level of either of these CARs on a single $\mathrm{T}$ cell would create a highly heterogenous pool of CAR T cells that may impinge on its tumor selectivity and ultimate potency. Furthermore, as with single-targeting CARs, the dual/multi-targeting CARs can also elicit selective pressure that could be stronger due to enhanced immune response, resulting in simultaneous escape of both antigens. Beyond technical complexities, another apparent 
challenge is the risk of high-grade CRS that comes with augmented $\mathrm{T}$-cell activation from concurrent antigen targeting ${ }^{75}$.

To ameliorate the toxicities arising from hyperactivated CAR $\mathrm{T}$ cells and on-target/off-tumor specificity, some CARs have been modified to contain an inhibitory/suicide gene, which when required, can be triggered to switch off T-cell signaling to avert ensuing toxicity. Suicide genes such as truncated EGFR (NCT03070327 and NCT03093168), iCasp9 (NCT03958656 and NCT03125577), and HSV-TK (NCT04097301) are currently being investigated in myeloma. Other safety strategies such as bispecific T-cell engager and tandem CAR have yet to be explored in myeloma and are therefore worth looking into ${ }^{20}$.

Thus far, generic CAR models consist of either CD28 or $4-1 \mathrm{BB}$ as co-stimulatory domain. The choice between these two molecules for CARs is essential as it determines the rapidity of immune activation and the persistence of the CAR T cells. CD28-harboring cells were shown to possess higher potency, rapid expansion, and ability to elicit quick cytokine surge following antigen stimulation, whereas, 41BB-containing CARs demonstrated lower signaling intensity, gradual activation, and a memory stem cell-like phenotype ${ }^{76}$. Although the former is associated with swift and robust cytolytic activities, its brisk signaling kinetics was associated with faster T-cell exhaustion, thus, resulting in less durable responses. They lack ongoing persistence compared to their 4-1BB counterpart, making them a likely inferior choice for a CAR construct ${ }^{13,66}$. The lesser preference for CD28-CAR in MM is evident (Table 2), whereby, after the first-in-human CD28-equipped BCMACAR $\mathrm{T}$ trial $(\mathrm{NCI})$, subsequent CAR $\mathrm{T}$ products predominantly carry 4-1BB.

Second-generation CARs are leading the pack in CAR T-cell research and investigation of the third- and fourthgeneration CARs in myeloma is scarce. Although the third-generation CARs should logically possess superior performance than its predecessor, most clinical data, at least in B-cell malignancies, demonstrated minimally augmented efficacy ${ }^{77}$. In MM, the efficacy of the thirdgeneration BCMA- and CD19-CAR constructs (CD28OX40-CD3 ) were tested by infusing the product cocktail into RRMM patients, post-ASCT. The ORR was $100 \%$, with low grade $\mathrm{CRS}^{54}$. This then begs the question of whether the response was truly attributable to the activity of third-generation CARs or was it an effect of dualantigen targeting with successful ASCT. A study making a parallel comparison between the second- and thirdgeneration CARs in a single experimental setup may be imperative. Likewise, the concept of fourth-generation CARs is well-received for its role in immunomodulation and reprogramming of tumor microenvironment; however, the knowledge on its efficacy compared to secondand third-generation CARs is limited by the lack of clinical pursue ${ }^{28}$. A search on the ClinicalTrial.gov registry's portal revealed 16 trials on fourth-generation CARs, two of which are for myeloma. These trials explore the efficacy of single or multi-antigen CAR T-cell compounds (BCMA, CD138, CD38, Integrin 37 ) (NCT03778346 and NCT03271632). While novel designs intended to increase CAR T-cell efficacy in myeloma are crucial, practical elements should also be considered, as higher innovations are often more labor-intensive and less cost-effective, not to mention the accompanying toxicity profile $^{28}$.

Short clinical remissions in MM have been attributed to low persistence of the BCMA CAR-T cells. To circumvent this, bb21217 that was derived from its precursor bb2121, was cultured ex vivo in the presence of PI3K inhibitor bb007, to enrich $\mathrm{T}$ cells with memory-like phenotype. The benefit of this approach was proven, when bb21217, but not bb2121, induced tumor clearance during a second tumor challenge within the same mice, without the need for re-administration of the product ${ }^{78}$. Bbb21217 displayed higher levels of CCR7 and CD27 compared to bb2121, suggesting higher levels of memory-like $\mathrm{T}$ cells, and lower levels of CD57, a marker of T-cell exhaustion. The latest update on bb21217 indicated 55\% ORR with the CAR T cells being detectable up to 18 months ${ }^{39,43}$. Additionally, the contribution of immunosuppressive factors in tumor microenvironment to the lack of durability of CAR-T cells is also notable. A new fully human BCMA-CAR harboring a dominant negative domain against the immunosuppressive TGF- $\beta$ was recently reported to exhibit robust proliferation and persistence, and remains functionally impervious to hostile TGF$\beta$-rich microenvironment ${ }^{79}$.

Another manufacturing optimization that has also been attempted to amplify the potency of CAR $\mathrm{T}$ cells in myeloma is the T-cell composition. A higher CD4:CD8 Tcell ratio utilized at the pre-transduction and expansion stage was associated with greater in vivo BCMA-targeted CAR T-cell expansion and response ${ }^{74}$, corroborating the reports in the CD19-targeted CAR T-cell therapy in lymphoma and ALL ${ }^{80,81}$. JCARH125 was infused into the RRMM patients (EVOLVE) with a designated CD4:CD8 ratio of 1:1. Latest clinical data reported robust expansion of these products at all dose levels, with patients' ORR $(82 \%)$ closely associated with the composite $\mathrm{T}$ cells ${ }^{82}$.

Gene delivery method is another variable in MM CAR T manufacturing, of which virus transduction is most conventionally opted. Given that this system comes with the risk of random insertional mutagenesis and is often associated with laborious and costly manufacturing procedures, other non-viral ways of introducing CARs into $\mathrm{T}$ cells have been introduced. The first non-viral CAR T-cell product for myeloma was developed by Poseida Therapeutics utilizing the piggyback-transposon 
technology (P-BCMA-101). This mRNA electroporation method demonstrated $>95 \%$ efficiency and a more stable and higher CAR expression, coupled with a prolonged activity from the expansion of $T$ cells with memory stem cell phenotype, when compared to those delivered by virus $^{74,83,84}$. Additionally, another non-viral system, the Sleeping Beauty, was used to introduce SLAMF7-CAR into the effector cells and has demonstrated good integration ${ }^{60}$.

Another CAR $\mathrm{T}$ manufacturing consideration is the origin of the moAb. One of the described therapeutic limitations of the otherwise successful CD19-targeted CAR T cells in lymphoma was T-cell fratricide due to host immune anti-murine CAR responses ${ }^{80}$. Integrating the less immunogenic humanized ScFVs into $\mathrm{T}$ cells is therefore a pragmatic approach. Indeed, the fully humanized BCMA-targeted CAR T cells (FCARH143 and JCARH125) developed by MSKCC exhibited rapid expansion, eradication of large tumor burden, and more durable patient response ${ }^{66,78}$. A novel, fully human, heavy-chain-only anti-BCMA binding domain (FHVH33) instead of a full ScFV, that was engineered to avert host immune cells' recognition, has been shown to induce deep and durable responses with manageable toxicities ${ }^{53}$.

\section{Perspectives}

The quest to identify novel targetable myeloma antigens is crucial; however, those that have been deliberated thus far did not seem able to uphold the potential of BCMA's. As a single agent, non-BCMA CAR $\mathrm{T}$ cells lacked meaningful outcomes. Refinements to these CARS such as rendering them bispecific often requires BCMA being the accompanying targeted antigen. With the impending FDA-approval of bb2121, studies on elucidating the underlying resistance mechanisms and ways of circumventing it, is indispensable.
Synthetic biology is crucial for engineering more potent BCMA-targeting CAR T cells, nevertheless, maintaining tumor-associated-BCMA expression and preventing antigen loss, is required for their execution of potency. In this instance, pharmacological means have been explored. $\gamma$ secretase inhibitor (GSI) treatment was able to increase BCMA density on tumor surface alongside decreased sBCMA concentration and improved CAR T-cell antitumor activity ${ }^{85,86}$. Oral administration of GSI with CAR $\mathrm{T}$ cells is currently being investigated in a Phase I trial (NCT03502577). Out of 10 evaluable patients, 30 and 50\% achieved sCR/CR and VGPR, respectively ${ }^{85,87}$. Interestingly, all-trans retinoic acid (ATRA), that was reported to rescue daratumumab sensitivity by increasing $\mathrm{CD} 38$ expression $^{88}$, was also found to be able to augment BCMA expression through an epigenetic modulation and enhances its recognition by BCMA-CAR T cells ${ }^{86}$. Combination treatment with ATRA and GSI led to higher BCMA density on MM cells, resulting in maximized reactivity. It is, again, imperative that we first clarify the inconclusive notion on the importance of baseline BCMA in conferring responsiveness. Not only is it necessary to ascertain the percentage of plasma cells having BCMA positivity, knowledge on the antigen density in each of the $\mathrm{BCMA}^{+}$ cells is also critical for discerning the BCMA threshold responsible for efficient CAR $\mathrm{T}$-cell recognition.

More recently, new attempts to leverage on the success of anti-CD19 CAR T therapies have emerged. Novel CD19-fusion protein models were developed to reactivate the targetability of relapsed NHL with low CD19 expression $^{89}$ and HER2-positive solid tumors ${ }^{90}$. Effective CD19-specific CAR T-cell-mediated cytotoxicity was observed in cases where artificial CD19 expression was successfully created on the surface of these CD19-low/ negative tumor cells. Such innovative strategy may also be considered for MM (Fig. 5). In this instance, a decoy CD19 antigen can be engineered on the surface of

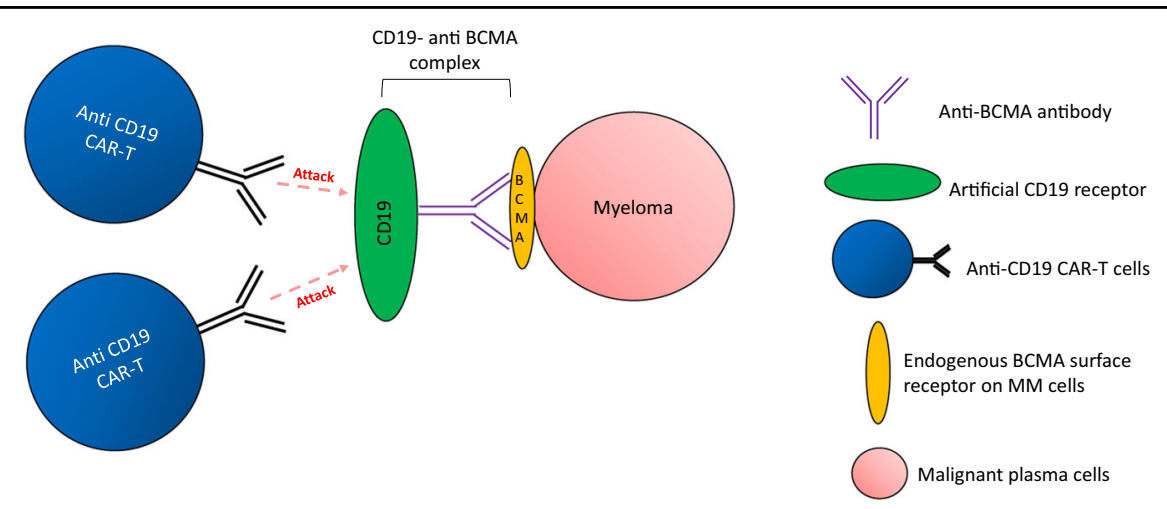

Fig. 5 CD19-antigen decoy for innovative targeting of myeloma cells. Increased expression of CD19 is induced on the myeloma cells by artificially creating the protein-anti-BCMA antibody complex that binds to endogenous surface BCMA. Highly potent and specific anti-CD19 CAR-T can be used to target these synthetic myeloma cells. 
myeloma cells by building a CD19-BCMA (or any other specific MM antigen) antibody complex, with the BCMA arm attaching to the tumor antigen. Overexpression of synthetic CD19 on MM would enable us to leverage on the already commercialized CD19-targeted CAR T cells (Kymriah or Yescarta) that are proven highly specific and potent.

Current standard-of-care has proven that combination of multiple agents targeting different mechanisms is the more effective way for disease management. However, investigations into BCMA CAR T therapy in combination with the diverse portfolio of anti-myeloma agents are rather limited. A preclinical work combining CAR T cells with lenalidomide found that the latter could enhance the persistence and activity of the former ${ }^{91,92}$. Another study reported that BCMA inhibition by the novel BCMAtargeting ADC (MEDI2228) could synergize with bortezomib in drug-resistant $\mathrm{MM}$ cells ${ }^{93}$. Various signaling inhibitors such as BRD4i and MEKi were shown to boost CAR $\mathrm{T}$ cell's functionality by hampering their immunosuppressive effects but these have never been studied in myeloma ${ }^{94,95}$. Plausibly in a drug combination study, one could investigate whether or not to incorporate an agent concomitantly with the CAR T-cell infusion or for maintenance post-CAR $T$ therapy, which may be useful for patients with huge MM burden.

The impressive results obtained thus far from CAR Tcell therapy trials in advanced and heavily pretreated MM patients have prompted the question of whether CAR $\mathrm{T}$ cells should be used as an earlier-line therapy. The lower disease burden in newly diagnosed or early-stage diseases may represent higher chance of achieving tumor clearance. In these cases, CARs with CD28, instead of 4$1 \mathrm{BB}$ co-stimulatory domain, may be a better choice as they elicit faster immune response. Swift activity of CD28equipped-CAR $\mathrm{T}$ cells would be useful for prompt therapeutic effects, thereby, better controlling the disease at an early stage, preventing progression. This would appear to be even more critical for high-risk patients such as double-hit or functional high-risk myeloma whose disease often advances quickly even with modern treatment strategy ${ }^{5}$. Attempt into investigating bb2121 in high-risk newly diagnosed patients has been initiated in KarMMa-4 trial (NCT04196491) ${ }^{96}$.

Additionally, myeloma has long been associated with quantitative and functional deficient $\mathrm{T}$ cells, particularly in the progressive, refractory disease. Acquisition of $\mathrm{T}$ cells from healthy donors is an attractive solution to the problem of insufficient and inferior CAR T cells. There are a few known allogenic compounds in MM that have gained IND clearance from the FDA, namely, UCARTCS1 (SLAMF7), CTX120 (BCMA), ALLO-715 (BCMA), CYAD-211 (BCMA), and PBCAR269A (BCMA) ${ }^{97,98}$. These products were conceived using different technologies including the non-gene editing method to eliminate TCR (CYAD-211) and single-step platform to concurrently knock-out TCR while knocking-in CAR $(\text { PBCAR269A })^{99}$. Another BCMA allogeneic compound (ALLO-605) that is being evaluated pre-clinically incorporates additional chimeric features to enhance cytokine signaling ${ }^{100}$. Besides addressing the T-cell quality, off-theshelf accessibility of these products also means that we can by-pass the long and complex autologous manufacturing process, thus, allowing for more rapid frontline therapies. The sustained availability of these allogenic CAR T cells will be useful for eradication of residual malignant cells, without the need for multiple rounds of painstaking autologous T-cell harvesting from the patients. In short, a cell bank with universal CAR $\mathrm{T}$ cells for allogeneic cell transfer would provide more flexibility in the application, faster delivery to the patients with less cost-intensive manufacturing.

Of late, CAR NK in myeloma is slowly gaining traction, owing to its better safety profile, versatility in its mechanisms of killing, and lower risk of GVHD, thereby higher feasibility for allogenic manufacturing that translates into cost effectiveness ${ }^{101}$. Several preclinical studies have demonstrated cytotoxic activity and MM growth inhibition using CAR NK cells against various targets, including CS1, CD138, BCMA, and NKG2D ligands ${ }^{102}$. There is currently one ongoing BCMA-CAR NK Phase $1 /$ 2 study in RRMM (NCT03940833). Furthermore, leveraging on the stem cells reprogramming system, an innovative CAR NK model, harboring a recombinant IL-15 signaling complex, was generated from induced pluripotent stem cells (iPSC). It has shown therapeutic efficacy alongside good synergism with moAb drugs (daratumumab, elotuzumab, and anti-CD19) ${ }^{103}$. With the reported safety properties of CAR NK cells over CAR $\mathrm{T}$ cells, we envision that the CAR NK therapy research field in MM will gain an incremental interest that will allow for the diversification of MM cell therapy product portfolio, although its potential drawbacks are also to be cautiously weighed.

On top of bio-engineering and diverse clinical testings, basic mechanistic research is also of importance as it provides insights into the fundamentals of immune response. Understanding the basic biology of $\mathrm{T}$ cells would enable us to produce 'fitter' CAR $\mathrm{T}$ cells that possess enhanced proliferation and longer endurance. Edging on the advancement of CRISPR-Cas9 technology, a genome-wide screening process can be performed to identify novel genes such when manipulated, could confer enhanced resilience and capabilities to the CAR T cells. Epigenetic mechanisms such as chromatin accessibility, DNA methylation states, RNA landscape in $T$ cells, immune profiling, and identification of immune checkpoint signature are some of the key areas for further 
investigation. Recoding the T-cell behavior via gene knock in/out approach would be helpful in addressing the issue of innate T-cell defects and inefficient pool of effector cells. To understand disease recurrence in a highly heterogeneous pool of MM cells, leveraging on the advent of single-cell sequencing technology may be able to help us obtain a high-level understanding of the temporal evolution on genetic and epigenetic aspects of the immune cells. A recent single-cell study of a plasma cell leukemia patient revealed distinct CAR T-cell subsets having different expression, proliferation, and cytotoxicity, indicating stage-specific changes along the developmental trajectory ${ }^{104}$. Considering this and MM heterogeneity, it would therefore be interesting to observe how different genomics and transcriptomics, such as different TC classes and RNA editing signatures, could dictate expression of surface targets that can be of use for immune targeting.

Beyond the basic biology, ex vivo and scaling processes also play a big part in CAR T-cell production ${ }^{37}$. Logistic factor involving time elapses between apheresis and delivery of manufactured CAR T cells back to patients is critical as the current turnaround time of 2-4 weeks renders CAR T-cell therapy unsuitable for patients with rapidly progressing disease. One emerging technology addressing this lengthy manufacturing issue is GraCell Bio's propriety FasT CAR platform, in which product release can be achieved within $22-36 \mathrm{~h}$ using an expedited protocol to activate and transduce $\mathrm{T}$ cells without the need for further ex vivo expansion. A dual BCMA-CD19 CAR T therapy was developed using this platform and the first-inhuman study has demonstrated an effective $93.8 \%$ ORR $^{105}$. Such finding should be a precursor to further identification of key bottlenecks along the whole CAR T-cell manufacturing pipeline (Fig. 4) and ways to optimize the steps to allow for less costly and cumbersome processes.

\section{Conclusion}

The CAR T-cell field in MM has indeed come a long way since the first BCMA-targeted CAR was developed in 2013. In our constant pursuit to develop personalized medicine, CAR T-cell therapy is perhaps the ultimate, as nothing can be more personalized than a treatment that harnesses the patient's own immune system for tumor destruction. BCMA has gained the 'holy grail' recognition for the CAR T-cell field in myeloma and as long as continuous effort is invested into circumventing the barriers that have limited the success of current BCMA-directed CAR T-cell products, we may soon be able to produce an optimized product for MM that could be within the same realm of success as CD19-targeting CARs in lymphomas and ALL. Although the emergence of novel drugs has brought about significant improvement in patients' outcome, these synthetic agents are physiologically eliminated from the body over time, making it difficult to produce a durable response without repetitive administration. CAR $\mathrm{T}$ cells, in constrast, is deemed a 'living drug' as they would be induced as long as targeted tumor antigen exists, thus making its therapeutic effect sustainable. If its efficacy is long-standing enough, we may well be on our way to having patients with lasting remission. With this and the anticipation for FDA's approval of bb2121 this year, we have every single reason to remain hopeful and optimistic that CAR T-cell therapies may one day render MM a chronic but highly manageable and curable disease.

\section{Acknowledgements}

We would like to thank Dr. Jennie Wong from National University of Singapore for proof reading the article.

\section{Author details}

${ }^{1}$ Department of Medicine, Yong Loo Lin School of Medicine, National University of Singapore, Singapore, Singapore. ${ }^{2}$ Cancer Science Institute of Singapore, Singapore, Singapore. ${ }^{3}$ Department of Haematology-Oncology, National University Cancer Institute of Singapore, National University Health System, Singapore, Singapore

\section{Author contributions}

P.J.T. performed literature search, determined the main content and designed the flow of this review, and completed the writing through its entirety. W.J.C. provided input, proofread, and finalized the manuscript.

Conflict of interest

The authors declare no competing interests.

\section{Publisher's note}

Springer Nature remains neutral with regard to jurisdictional claims in published maps and institutional affiliations.

Received: 1 December 2020 Revised: 16 March 2021 Accepted: 7 April 2021 Published online: 29 April 2021

\section{References}

1. Robak, P. \& Robak, T. Bortezomib for the treatment of hematologic malignancies: 15 years later. Drugs in R\&D 19, 73-92 (2019).

2. Kumar, S. K. et al. Safety and tolerability of ixazomib, an oral proteasome inhibitor, in combination with lenalidomide and dexamethasone in patients with previously untreated multiple myeloma: an open-label phase 1/2 study. Lancet Oncol. 15, 1503-12 (2014).

3. Timmers, M. et al. Chimeric antigen receptor-modified $\mathrm{T}$ cell therapy in multiple myeloma: beyond B cell maturation antigen. Front. Immunol. 10, 1613- (2019).

4. Dingli, D. et al. Therapy for relapsed multiple myeloma: guidelines from the Mayo stratification for myeloma and risk-adapted therapy. Mayo Clinic Proc. 92, 578-98 (2017).

5. Walker, B. A. et al. A high-risk, double-hit, group of newly diagnosed myeloma identified by genomic analysis. Leukemia. 33, 159-70 (2019).

6. Kazmi, S. M. et al. Outcomes among high-risk and standard-risk multiple myeloma patients treated with high-dose chemotherapy and autologous hematopoietic stem-cell transplantation.Clin. Lymphoma Myeloma Leuk. 15, 687-93 (2015).

7. Chng, W. J. et al. IMWG consensus on risk stratification in multiple myeloma. Leukemia 28, 269-77 (2014).

8. Guedan, S., Ruella, M. \& June, C. H. Emerging cellular therapies for cancer. Annu. Rev. Immunol. 37, 145-71 (2019).

9. Lei, M. et al. Current management and emerging treatment strategies for multiple myeloma. Jpn. J. Clin. Hematol. 60, 1243-56 (2019). 
10. Chmielewski, M. et al. T cells that target carcinoembryonic antigen eradicate orthotopic pancreatic carcinomas without inducing autoimmune colitis in mice. Gastroenterology. 143, 1095-107.e2 (2012).

11. Kalos, M. et al. T cells with chimeric antigen receptors have potent antitumor effects and can establish memory in patients with advanced leukemia. Sci. Transl. Med. 3, 95ra73-95ra73 (2011).

12. Kohl, U., Arsenieva, S., Holzinger, A. \& Abken, H. CAR T Cells in trials: recent achievements and challenges that remain in the production of modified T cells for clinical applications. Hum. Gene Ther. 29, 559-68 (2018).

13. Ormhøj, M., Bedoya, F., Frigault, M. J. \& Maus, M. V. CARs in the lead against multiple myeloma. Curr. Hematol. Malig. Rep. 12, 119-25 (2017).

14. Charrot, S. \& Hallam, S. CAR-T cells: future perspectives. Hemasphere 3, e188-e (2019).

15. Turtle, C. J. et al. CD19 CAR-T cells of defined CD4+:CD8+ composition in adult B cell ALL patients. J. Clin. investig. 126, 2123-38 (2016).

16. Abramson, J. S. et al. Lisocabtagene maraleucel for patients with relapsed or refractory large B-cell lymphomas (TRANSCEND NHL 001): a multicentre seamless design study. Lancet 396, 839-52 (2020).

17. Eshhar, Z., Waks, T., Gross, G. \& Schindler, D. G. Specific activation and targeting of cytotoxic lymphocytes through chimeric single chains consisting of antibody-binding domains and the gamma or zeta subunits of the immunoglobulin and T-cell receptors. Proc. Natl Acad. Sci. USA 90, 720 (1993).

18. Brentjens, R. J. et al. Eradication of systemic B-cell tumors by genetically targeted human T lymphocytes co-stimulated by CD80 and interleukin-15. Nat. Med. 9, 279-86 (2003).

19. Hollyman, D. et al. Manufacturing validation of biologically functional T cells targeted to CD19 antigen for autologous adoptive cell therapy. J. Immunother. 32, 169-80 (2009).

20. Bonifant, C. L., Jackson, H. J., Brentjens, R. J. \& Curran, K. J. Toxicity and management in CAR T-cell therapy. Mol. Ther. Oncolytics 3, 16011 (2016).

21. Holzinger, A. \& Abken, H. CAR T cells: a snapshot on the growing options to design a CAR. Hemasphere 3, 1 (2019).

22. Wang, M., Yin, B., Wang, H. Y. \& Wang, R. F. Current advances in T-cell-based cancer immunotherapy. Immunotherapy 6, 1265-78 (2014).

23. Watanabe, K., Kuramitsu, S., Posey, A. D. Jr. \& June, C. H. Expanding the therapeutic window for CAR T cell therapy in solid tumors: the knowns and unknowns of CAR T cell biology. Front. Immunol. 9, 2486- (2018).

24. Long, A. H. et al. 4-1BB costimulation ameliorates T cell exhaustion induced by tonic signaling of chimeric antigen receptors. Nat. Med. 21, 581-90 (2015)

25. Milone, M. C. et al. Chimeric receptors containing CD137 signal transduction domains mediate enhanced survival of $T$ cells and increased antileukemic efficacy in vivo. Mol. Ther. 17, 1453-64 (2009).

26. Philipson, B. I. et al. 4-1BB costimulation promotes CAR T cell survival through noncanonical NF-kappaB signaling. Sci. Signal. 13, 625 (2020).

27. Krause, A. et al. Antigen-dependent CD28 signaling selectively enhances survival and proliferation in genetically modified activated human primary $\mathrm{T}$ lymphocytes. J. Experimental Med. 188, 619-26 (1998).

28. Chmielewski, M., Hombach, A. A. \& Abken, H. Of CARs and TRUCKs: chimeric antigen receptor (CAR) T cells engineered with an inducible cytokine to modulate the tumor stroma. Immunol. Rev. 257, 83-90 (2014).

29. Chmielewski, M., Kopecky, C., Hombach, A. A. \& Abken, H. IL-12 release by engineered $\mathrm{T}$ cells expressing chimeric antigen receptors can effectively Muster an antigen-independent macrophage response on tumor cells that have shut down tumor antigen expression. Cancer Res. 71, 5697-706 (2011).

30. Wang, X. \& Rivière, I. Clinical manufacturing of CAR T cells: foundation of a promising therapy. Mol. Ther. 3, 16015 (2016).

31. Carpenter, R. O. et al. B-cell maturation antigen is a promising target for adoptive T-cell therapy of multiple myeloma.Clin. Cancer Res. 19, 2048-60 (2013).

32. Seckinger, A. et al. Target expression, generation, preclinical activity, and pharmacokinetics of the BCMA-T cell bispecific antibody EM801 for multiple myeloma treatment. Cancer Cell 31, 396-410 (2017).

33. Friedman, K. M. et al. Effective targeting of multiple B-cell maturation antigen-expressing hematological malignances by anti-B-cell maturation antigen chimeric antigen receptor T cells. Human Gene Ther. 29 585-601 (2018)

34. Moreaux, J. et al. The level of TACl gene expression in myeloma cells is associated with a signature of microenvironment dependence versus a plasmablastic signature. Blood 106, 1021-30 (2005).
35. Tai, Y.-T. et al. APRIL and BCMA promote human multiple myeloma growth and immunosuppression in the bone marrow microenvironment. Blood 127, 3225-36 (2016).

36. Ghermezi, M. et al. Serum B-cell maturation antigen: a novel biomarker to predict outcomes for multiple myeloma patients. Haematologica $\mathbf{1 0 2}$ 785-95 (2017).

37. Ali, S. A. et al. T cells expressing an anti-B-cell maturation antigen chimeric antigen receptor cause remissions of multiple myeloma. Blood 128, 1688-700 (2016)

38. Brudno, J. N. et al. T cells genetically modified to express an anti-B-cell maturation antigen chimeric antigen receptor cause remissions of poorprognosis relapsed multiple myeloma. J. Clin. Oncol. 36, 2267-80 (2018).

39. Raje, N. et al. Anti-BCMA CAR T-cell therapy bb2121 in relapsed or refractory multiple myeloma. New Engl. J. Med. 380, 1726-37 (2019).

40. Shah, N. et al. Initial results from a Phase 1 clinical study of bb21217, a nextgeneration anti Bcma CAR T therapy. Blood 132, 488- (2018).

41. Jagannath, S. et al. KarMMa-RW: a study of real-world treatment patterns in heavily pretreated patients with relapsed and refractory multiple myeloma (RRMM) and comparison of outcomes to KarMMa. J. Clin. Oncol. 38, 8525 (2020).

42. Munshi, N. C. et al. Idecabtagene Vicleucel in relapsed and refractory multiple myeloma. New Engl. J. Med. 384, 705-16 (2021).

43. Finney, O. C. et al. Molecular and phenotypic profiling of drug product and post-infusion samples from CRB-402, an ongoing: Phase I clinical study of bb21217 a BCMA-directed CAR T cell therapy. Blood 136, 3-4 (2020).

44. Madduri, D. et al. Results from CARTITUDE-1: A Phase 1b/2 study of JNJ-4528, a CAR-T cell therapy directed against B-Cell Maturation Antigen (BCMA), in patients with Relapsed and/or Refractory Multiple Myeloma (R/R MM). Blood. 134, 577- (2019).

45. Madduri, D. et al. CARTITUDE-1: Phase $1 \mathrm{~b} / 2$ study of Ciltacabtagene Autoleucel, a B-cell maturation antigen-directed chimeric antigen receptor $\mathrm{T}$ cell therapy, in relapsed/refractory multiple myeloma. Blood 136, 22-5 (2020).

46. Wang, B. et al. Chimeric antigen receptor T cell therapy in the relapsed or refractory multiple myeloma with extramedullary disease-a single institution observation in China. Blood. 136, 6- (2020).

47. Shah, N., Chari, A., Scott, E., Mezzi, K. \& Usmani, S. Z. B-cell maturation antigen $(B C M A)$ in multiple myeloma: rationale for targeting and current therapeutic approaches. Leukemia 34, 985-1005 (2020).

48. Colonna, L. et al. Orvacabtagene Autoleucel (orva-cel; JCARH125): a fully human BCMA-targeted second-generation CAR T cell product characterized by a predominant central memory phenotype with high in vitro and in vivo proliferative potential and sustained in vivo persistence. Blood 136, 11-2 (2020)

49. Roex, G. et al. Safety and clinical efficacy of BCMA CAR-T-cell therapy in multiple myeloma. J. Hematol. Oncol. 13, 164- (2020).

50. Steiner, N. \& Gunsilius, E. CAR-T cells in multiple myeloma: current status. Memo 13, 43-9 (2020).

51. Da Vià M. C. et al. Homozygous BCMA gene deletion in response to antiBCMA CAR T cells in a patient with multiple myeloma. Nat. Med. 27, 616-619 (2021).

52. Samur, M. K. et al. Biallelic loss of BCMA triggers resistance to anti-BCMA CAR T cell therapy in multiple myeloma. Blood 136, 14 (2020).

53. Mikkilineni, $L$. et al. T cells expressing an anti-B-Cell Maturation Antigen (BCMA) chimeric antigen receptor with a fully-human heavy-chain-only antigen recognition domain induce remissions in patients with relapsed multiple myeloma. Blood. 134, 3230 (2019)

54. Lin, Q., Zhao, J., Song, Y. \& Liu, D. Recent updates on CAR T clinical trials for multiple myeloma. Mol. Cancer 18, 154 (2019).

55. Sanchez, E. et al. Serum B-cell maturation antigen is elevated in multiple myeloma and correlates with disease status and survival. Br. J. Haematol. 158 727-738 (2012).

56. Piasecki, J. et al. Association of baseline and postinfusion biomarkers with safety and efficacy endpoints in patients treated with Orvacabtagene Autoleucel (orva-cel; JCARH125) in the Phase $1 / 2$ evolve study (NCT03430011). Blood. 136, 2-3 (2020).

57. Dimopoulos, M. A. et al. Elotuzumab plus pomalidomide and dexamethasone for multiple myeloma. New Engl. J. Med. 379, 1811-22 (2018).

58. Lonial, S. et al. Elotuzumab therapy for relapsed or refractory multiple myeloma. New Engl. J. Med. 373, 621-31 (2015). 
59. Gogishvili, T. et al. SLAMF7-CAR T cells eliminate myeloma and confer selective fratricide of SLAMF7(+) normal lymphocytes. Blood. 130, 2838-2847 (2017).

60. Miskey, C. et al. Genomic analyses of SLAMF7 CAR-T cells manufactured by sleeping beauty transposon gene transfer for immunotherapy of multiple myeloma. Preprint at bioRxiv https://doi.org/10.1101/675009 (2019).

61. Mathur, R. et al. Universal SLAMF7-specific CAR T-cells as treatment for multiple myeloma. Blood. 130, 502 (2017).

62. Zah, E. et al. Systematically optimized BCMA/CS1 bispecific CAR-T cells robustly control heterogeneous multiple myeloma. Nat. Commun. 11, 2283 (2020).

63. Usmani, S. Z. et al. Daratumumab monotherapy in patients with heavily pretreated relapsed or refractory multiple myeloma: final results from the phase 2 GEN501 and SIRIUS trials. Lancet Haematol. 7, e447-e55 (2020).

64. Lonial, S. et al. Daratumumab monotherapy in patients with treatmentrefractory multiple myeloma (SIRIUS): an open-label, randomised, phase 2 trial. Lancet 387, 1551-1560 (2016).

65. Martin, T. et al. A phase $1 \mathrm{~b}$ study of isatuximab plus lenalidomide and dexamethasone for relapsed/refractory multiple myeloma. Blood 129, 3294-3303 (2017).

66. Ghosh, A. et al. CAR T cell therapy for multiple myeloma: where are we now and where are we headed? Leuk. Lymphoma 59, 2056-2067 (2018).

67. Drent, E. et al. Pre-clinical evaluation of CD38 chimeric antigen receptor engineered $T$ cells for the treatment of multiple myeloma. Haematologica 101, 616-25 (2016).

68. Li, C. et al. A bispecific CAR-T cell therapy targeting Bcma and CD38 for relapsed/refractory multiple myeloma: updated results from a Phase 1 doseclimbing trial. Blood 134, 930 (2019).

69. Lee, L. et al. An APRIL-based chimeric antigen receptor for dual targeting of BCMA and $\mathrm{TACl}$ in multiple myeloma. Blood 131, 746-58 (2018).

70. Schmidts, A. et al. Rational design of a trimeric APRIL-based CAR-binding domain enables efficient targeting of multiple myeloma. Blood Adv. $\mathbf{3}$, 3248-3260 (2019).

71. Larson, R. et al. Bispecific CAR T cells for multiple myeloma: natural ligand compared to tandem scFv design. J. Immunol. 204, 246.3 (2020).

72. Tai, Y.-T. et al. APRIL signaling via TACl mediates immunosuppression by T regulatory cells in multiple myeloma: therapeutic implications. Leukemia 33 426-438 (2019).

73. Mishra, A. K., Kemler, I. \& Dingli, D. Development of CAR-T cell constructs with broad anti-tumor tropism. Blood 136, 24 (2020)

74. Cohen, A. D. et al. B cell maturation antigen-specific CAR T cells are clinically active in multiple myeloma. J. Clin. Investig. 129, 2210-2221 (2019).

75. Cronk, R. J., Zurko, J. \& Shah, N. N. Bispecific chimeric antigen receptor T cell therapy for B cell malignancies and multiple myeloma. Cancers 12, 2523 (2020).

76. Abate-Daga, D. \& Davila, M. L. CAR models: next-generation CAR modifications for enhanced T-cell function. Mol. Ther. 3, 16014 (2016).

77. Gao, Q. et al. Therapeutic potential of CRISPR/Cas9 gene editing in engineered T-cell therapy.Cancer Med. 8, 4254-4264 (2019).

78. Feinberg, D., Paul, B. \& Kang, Y. The promise of chimeric antigen receptor (CAR) T cell therapy in multiple myeloma. Cellular Immunol. 345, 103964 (2019).

79. Alabanza, L. et al. A fully-human armored BCMA CAR boosts function of CD4+ CAR-T cells and resists TGF- $\beta$ suppression in pre-clinical models of multiple myeloma. Blood 136, 37-38 (2020).

80. Turtle, C. J. et al. Immunotherapy of non-Hodgkin's lymphoma with a defined ratio of CD8+ and CD4+ CD19-specific chimeric antigen receptor-modified T cells. Sci. Transl. Med. 8, 355ra116 (2016).

81. Yang, Y. et al. CD4 CAR T cells mediate CD8-like cytotoxic anti-leukemic effects resulting in leukemic clearance and are less susceptible to attenuation by endogenous TCR activation than CD8 CAR T cells. Blood 126, 100 (2015).

82. Mailankody, S. et al. JCARH125, anti-BCMA CAR T-cell therapy for relapsed/ refractory multiple myeloma: initial proof of concept results from a Phase 1/2 multicenter study (EVOLVE). Blood 132, 957 (2018).
83. Hermanson, D. L. et al. A novel Bcma-specific, centyrin-based CAR-T product for the treatment of multiple myeloma. Blood 128, 2127 (2016).

84. Costello, C. L. et al. Phase 2 study of the response and safety of P-Bcma-101 CAR-T cells in patients with Relapsed/Refractory ( $r / r)$ Multiple Myeloma (MM) (PRIME). Blood 134, 3184 (2019).

85. Pont, M. J. et al. $Y$-secretase inhibition increases efficacy of BCMA-specific chimeric antigen receptor T cells in multiple myeloma. Blood 134, 1585-97 (2019).

86. Garcia-Guerrero, E. et al. ATRA augments BCMA expression on myeloma cells and enhances recognition by BCMA-CAR T-cells. Blood 136, 13-14 (2020).

87. Green, D. J. et al. Response to Bcma CAR-T cells correlates with pretreatment target antigen density and is improved by small molecule inhibition of gamma secretase. Blood. 134, 1856 (2019).

88. Nijhof, I. S. et al. CD38 expression and complement inhibitors affect response and resistance to daratumumab therapy in myeloma. Blood 128, 959-970 (2016).

89. Rennert, P. et al. A novel CD19-anti-CD20 bridging protein prevents and reverses CD19-negative relapse from CAR19 T cell treatment in vivo. Blood 134, 252 (2019).

90. Ambrose, C. et al. Anti-CD19 CAR T cells potently redirected to kill solid tumor cells. PLOS ONE 16, e0247701 (2021).

91. Works, M. et al. Anti-B-cell maturation antigen chimeric antigen receptor $\mathrm{T}$ cell function against multiple myeloma is enhanced in the presence of lenalidomide. Mol. Cancer Ther. 18, 2246-2257 (2019).

92. Wang, $X$. et al. Lenalidomide enhances the function of CS1 chimeric antigen receptor-redirected T cells against multiple myeloma.Clin. Cancer Res. 24, 106-119 (2018).

93. Xing, L. et al. A novel BCMA PBD-ADC with ATM/ATRMEE1 inhibitors or bortezomib induce synergistic lethality in multiple myeloma. Leukemia $\mathbf{3 4}$ 2150-2162 (2020).

94. Kagoya, Y. et al. BET bromodomain inhibition enhances $T$ cell persistence and function in adoptive immunotherapy models. J. Clin. Investig. 126 3479-3494 (2016).

95. Gargett, T., Fraser, C. K., Dotti, G., Yvon, E. S. \& Brown, M. P. BRAF and MEK inhibition variably affect GD2-specific chimeric antigen receptor (CAR) T-cell function in vitro. J. Immunother. 38, 12-23 (2015).

96. Usmani, S. Z. et al. KarMMa-4: Idecabtagene Vicleucel (ide-cel, bb2121), a BCMA-Directed CAR T-cell therapy, in high-risk newly diagnosed multiple myeloma. Blood 136, 18-19 (2020).

97. Depil, S., Duchateau, P., Grupp, S. A., Mufti, G. \& Poirot, L. 'Off-the-shelf' allogeneic CAR T cells: development and challenges. Nat. Rev. Drug Discov. 19, 185-199 (2020)

98. Dar, H. et al. Preclinical development of CTX120, an allogeneic CAR-T cell targeting Bcma. Blood 132, 1921 (2018).

99. Al-Homsi, A. S. et al. Clinical development of a non-gene-edited allogeneic Bcma-targeting CAR T-cell product in relapsed or refractory multiple myeloma. Blood. 136, 27-28 (2020).

100. Sommer, C. et al. Preclinical evaluation of ALLO-605, an allogeneic BCMA turbocar TTM cell therapy for the treatment of multiple myeloma. Blood 136, 8 (2020).

101. Xie, G. et al. CAR-NK cells: a promising cellular immunotherapy for cancer. EBioMedicine 59, 102975 (2020)

102. Wang, W., Jiang, J. \& Wu, C. CAR-NK for tumor immunotherapy: clinical transformation and future prospects. Cancer Lett. 472, 175-180 (2020).

103. Goodridge, J. P. et al. FT576: multi-specific off-the-shelf CAR-NK cell therapy engineered for enhanced persistence, avoidance of self-fratricide and optimized mab combination therapy to prevent antigenic escape and elicit a deep and durable response in multiple myeloma. Blood 136, 4-5 (2020).

104. Li, X. et al. Single-cell transcriptomic analysis reveals BCMA CAR-T cell dynamics in a patient with refractory primary plasma cell leukemia. Mol. Ther 29, 645-657 (2021)

105. Jiang, $H$. et al. Clinical results of a multicenter study of the first-inhuman dual BCMA and CD19 targeted novel platform fast CAR-T cell therapy for patients with relapsed/refractory multiple myeloma. Blood 136, 25-26 (2020). 